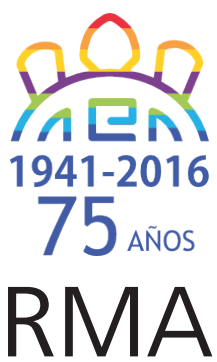

Arqueología

\title{
Construyendo preguntas en el camino. Comunidades originarias y científicas
}

\author{
Building questions along the way. Native and scientific \\ communities
}

Ricardo A. Guichón*

*CONICET, Núcleo de Estudios Interdisciplinarios de Poblaciones Humanas de Patagonia Austral (NEIPHPA), Laboratorio de Ecología Evolutiva Humana (Quequén), Facultad de Ciencias Sociales, Universidad Nacional del Centro de la Provincia de Buenos Aires, Argentina. E-mail:guichon2012@gmail.com

\begin{abstract}
Resumen
Más allá de las particularidades y experiencias que cada uno pueda tener en la relación entre comunidades originarias y comunidades de investigadores lo que aquí plantearemos es la posibilidad explorar nuestro "hacer-pensar" en términos de Najmanovich. Nuestro análisis estará guidado por el diálogo entre algunas experiencias personales y lecturas fuera del ámbito arqueológico "tradicional". Se trata de un espacio exploratorio donde Lederach trajo su perspectiva sobre los "conflictos sociales complejos"; Nato y colaboradores aspectos interesantes surgidos desde la "mediación comunitaria"; y por último Morín, Capra, García y Najmanovich sus perspectivas sobre "complejidad". Nuestra travesía se inicia con lo la dinámica del Modelo Lineal de la relación entre comunidades originarias (CO) y científicas (CI). El siguiente paso nos llevará a la judicialización y otras formas de abordar conflictos. Luego avanzaremos sobre posibles actitudes frente al conflicto. Aquí ingresaremos en un modelo no lineal de la relación entre CO y Cl. Todo este recorrido para llegar a algo en construcción espacios de diálogo.
\end{abstract}

Palabras clave: Bioantropologia; Patagonia; Conflictos; Perspectivas; Complejidad.

\begin{abstract}
Aside from the experiences and attitudes that each individual might have towards the relationship between indigenous communities and researchers, we present the need to rethink the "scenarios" where we are interact. Our analysis will be guided by the dialogue between personal experiences and ideas brought by specific authors outside the archaeological literature. This is an analysis, where Lederach's perspective on the "complex social conflicts" is explored; the" community mediation" of Nato and coauthors is introduced and finally Morin, Capra, Garcia and Najmanovich gave their perspectives on "complexity". Our journey begins with what we call linear model of the relationship between indigenous (CO) and scientific (CI) communities. The next step takes us to the "prosecution" and other ways of dealing with conflicts. Then we advance through the different attitudes towards the conflict. Here we enter into a nonlinear model of the relationship between $\mathrm{CO}$ and $\mathrm{Cl}$. This journey to get to something called "Building spaces for dialogue".
\end{abstract}

Keywords: Bio-Anthropology; Patagonia; Conflicts; Perspectives; Complexity.

En la diversidad de formas de relación entre miembros de comunidades originarias $(\mathrm{CO})$ e investigadores $(\mathrm{Cl})^{1}$ están presentes las experiencias e historias previas de cada lugar, las cosmovisiones y las cambiantes situaciones particulares (Najmanovich 2007). Por estas razones y porque todos los seres humanos nos caracterizamos, en

\footnotetext{
${ }^{1}$ Recuperamos la idea de comunidades pensando en personas que se reconocen como parte de un grupo que comparten un rango de actividades y perspectivas sobre las cuales hay cierto grado de consenso (explícitamente o no) en un momento y espacio acotado. Estas personas se relacionan con el resto de su sociedad con una variedad de estilos de interpretar y llevar adelante determinados marcos o encuadres. Pensar en comunidades científicas y originarias en un determinado espacio y tiempo (Local siguiendo a Geertz 1994) requiere de considerar tanto la variedad de actividades y formas de pensar que cada una desarrolla como también el conjunto de maneras que despliegan para relacionarse entre ellas y con otras comunidades.
}

términos generales, por la capacidad de responder de diferentes maneras ante un mismo problema (plasticidad conductual siguiendo a Maturana y Varela (1993) los análisis "simplistas" no son la única estrategia para pensar y actuar en estos contextos.

Más allá de las particularidades y experiencias que cada uno de nosotros pueda aportar lo que aquí planteamos es la posibilidad de algo así como plataformas ${ }^{2}$ orientadas a repensar los escenarios donde estamos actuando. Nos preguntamos si la lógica de la simplicidad es una de las posibilidades y si no será posible generar y caminar otras

\footnotetext{
2 Nos referimos a "plataforma" como espacios flexible, pensado o preparado para ser modificado y cambiado retomando la idea de Lederach (2006) sobre el término.
} 
de manera no lineal (Najmanovich 2002).

Lo que algunos autores denominan la perspectiva de complejidad podría encuadrarlos problemas de relaciones entre los miembros de $\mathrm{CO}$ y $\mathrm{Cl}^{3}$. Nuestro análisis en estas páginas estará guidado por el diálogo entre algunas experiencias personales y lecturas que aportaron ideas. Se trata de un espacio exploratorio donde Lederach (2006) colaboró con su mirada y perspectiva sobre los "conflictos sociales complejos"; Nato et al. (2006) trajo aspectos interesantes surgidos desde la "mediación comunitaria"; y por último Morín (2002), Capra (1996) García (2006) y Najmanovich (2002) sus perspectivas sobre "complejidad".

En trabajos previos, conjuntamente con el resto del grupo de trabajo, hemos planteado una interpretación sobre el contexto histórico en Patagonia Austral (ver Guichón y colaboradores 2016) y su formulación escapa a los objetivos de este trabajo. Recientemente el Ministerio de Educación de la Nación ha publicado un interesante trabajo donde se consideran las comunidades originarias actuales de Santa Cruz y Tierra del Fuego y como dejaron de ser "invisibles". Asimismo presentan sintéticamente los procesos de colonización en la región Patagónica Austral, cuyas consecuencias llegan hasta el presente. En tercer lugar detallan cómo fueron descriptos y estudiadas las comunidades originarias a comienzos del siglo XX y por último hace referencia a sus luchas, deseos y demandas actuales (Rodríguez y colaboradores 2016).

\footnotetext{
${ }^{3}$ Aquí cuando nos referimos a comunidades de investigadores (Cl) es posible abrir otras "ventanas" como discuten Restrepo y Escobar (2005) repensando la pluralidad de prácticas profesionales y las tensiones que existen y existieron en las maneras de hacer antropología dentro de las mismas. Entendemos que estamos explorando en "otras maneras" de hacer Antropología vinculable a los autores antes mencionados y los planteos de Rappaport (2007).
}

Cabe aclarar que las denominadas experiencias "personales" de las que hablamos son el resultado de intercambios con otras personas con las cuales construimos vínculos a partir de los cuales aprendimos y aprendemos. Alguien podría preguntar "y en la práctica que ocurrió". Por ejemplo en Puerto Santa Cruz con la Comunidad Lof Fem Mapu surgieron dos instrumentos nuevos en el ámbito municipal denominados "Reservorio Cogestionado Transitorio" y "Comisión de cuidado del Pasado". Ambos toman forma en la ordenanza Municipal de Puerto Santa Cruz 169 del 2009. No vamos aquí a referirnos a los detalles de la ordenanza (ver Nahuelquir y colaboradores 2015) pero sí señalar algunos aspectos que pueden ayudarnos. De común acuerdo entre todos los actores interesados (comunidades, autoridades municipales e investigadores que trabajan la zona) se buscó un espacio cogestionado transitorio donde se ubicaron todos los restos óseos humanos resultantes de hallazgos sistemáticos (realizados por arqueólogos sobre la base de consensos técnico metodológicos) y asistemáticos (realizados por pobladores locales). Luego de muchas charlas entre los actores antes mencionados acordamos, entre otras cosas, que se trataba de restos de personas muertas y no de objetos de colección, y que debían tener un trato especial. Estaba clara la existencia de al menos dos visiones (la de la comunidad y la del investigador científico), sin embargo encontramos que era posible generar una situación transitoria y cogestionada. En el reservorio una de las llaves está en manos de la Comunidad Lof Fem Mapu (CLFM) y la otra en manos de representes de la Municipalidad. Es transitorio dado que de acuerdo a la Ley las comunidades originarias pueden reclamarlos restos ${ }^{4}$.

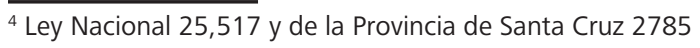

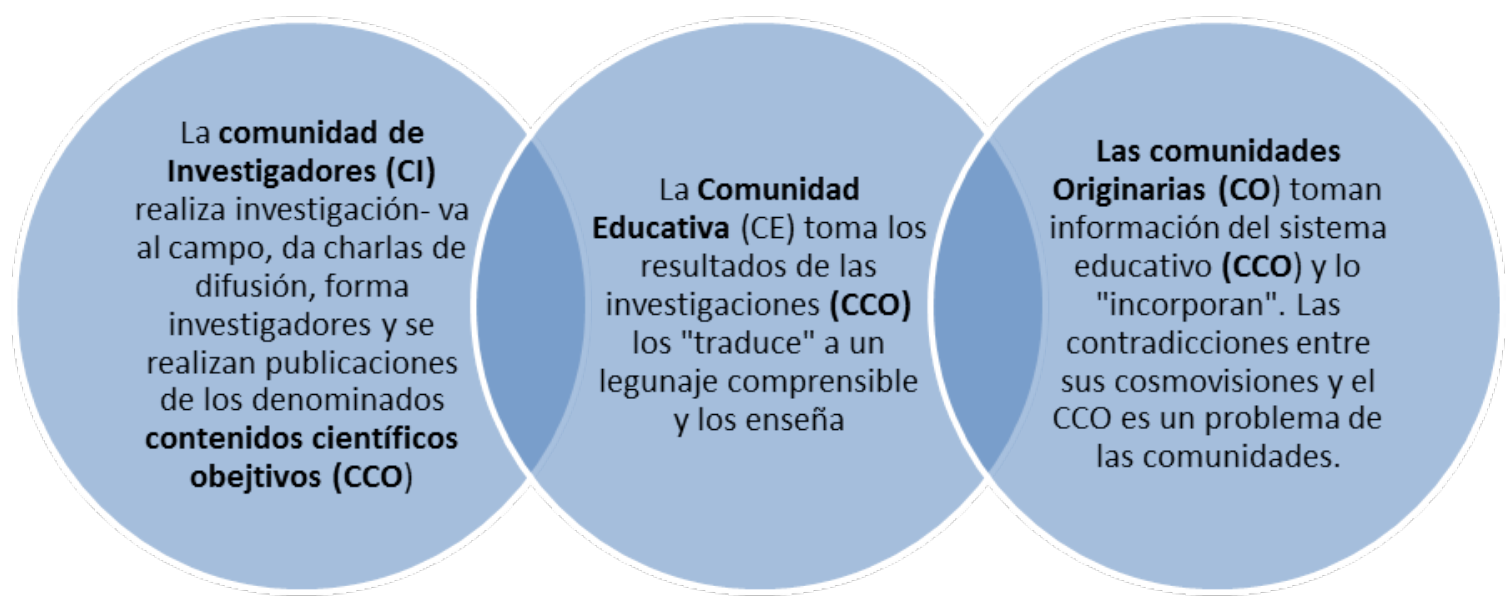

Figura 1: Relación entre Comunidades (Investigadores, Educativa y Originarias). Modelo lineal simple

Figure 1: Relationship between Communities (Researchers, Education and Native) Simple linear model. 
Nuestra travesía se inicia con lo que llamaremos Modelo Lineal de la relación entre comunidades originarias (CO) y científicas (Cl). El siguiente paso nos llevará a la judicialización y otras formas de abordar los conflictos. Luego avanzaremos sobre posibles actitudes frente al conflicto. Aquí ingresaremos en un modelo no lineal de la relación entre $\mathrm{CO}$ y $\mathrm{Cl}$. Un pequeño desvió nos llevara a recorrer algunas ideas sobre interdisciplina. Todo este recorrido para llegar de una especie de "plataforma" en construcción aún y que llamamos espacios de diálogo y encuadre. Por último presentaremos una agenda abierta orientada a redefinir la travesía.

\section{Modelo lineal de relación entre $\mathrm{CO}$ y $\mathrm{Cl}$}

Nuestra experiencia surge del encuentro de personas (miembros CO y de Cl) motivadas por diferentes intereses. Además de trabajar en Puerto Santa Cruz también lo hago en Tierra del Fuego como investigador especialista en el estudio de restos humanos y director de un grupo de investigación ${ }^{5}$ (Guichón y colaboradores 2015). Allí nos propusimos investigar los cambios en salud que generó el proceso de contacto europeo indígena. Nuestro proyecto se planteó la excavación del cementerio de la Misión Salesiana Nuestra Señora de la Candelaria en Río Grande. Cabe señalar que en el 2006 se inició el proyecto gracias al apoyo del MINCyT y a las consultas previas con todos los actores interesados además de los permisos oficiales. Actualmente se están analizando restos humanos de miembros de la comunidad Selk'nam ${ }^{6}$, cómo de los denominados "antiguos pobladores de la zona", y de Hermanas de María Auxiladora ${ }^{8}$. Aquí no hay "recetas": las experiencias concretas de las que hablamos continúan siendo espacios de aprendizaje. Sin embargo pese a que todo lo que aquí presentamos está pensando en relación a actores y circunstancias concretas esto no quiere decir que este camino no pueda ser útil en otros contextos.

Luego de esta de presentación de nuestros ámbitos de acción vamos a explicitar la dinámica de lo que denominamos Modelo Lineal de relación entre CO y Cl (ver

\footnotetext{
5 Nuestro grupo de investigación (NEIPHPA-LEEH-FACSO-UNCPBA) está integrado por personas con trayectorias y experiencias de trabajos previos diversas. Integrar/nos y relacionar/nos desde diferentes marcos de referencia teóricos y metodológicos (Arqueólogos, Biólogos, Historiadores y biontropologos) es uno de los desafíoslo conforman. Cómo señala Morín (1993) la totalidad, además de poder ser la suma de las partes o más que ella, también puede ser menos. Hay que considerar también aspectos que pueden inhibir o potenciar.

${ }^{6}$ La identidad Nativo Americano se está realizando por medio de estudios de ADN mitocondrial. Más de la mitad (sobre un total de 30 personas exhumadas) ya fueron estudiadas. Los haplogrupos identificados corresponden a los esperados para la zona. Además se realizaron estudios de isótopos estables para identificar donde consumieron agua cuando eran pequeños y en los últimos años de vida además de la dieta.

${ }^{7}$ Por los mismos métodos mencionados en el pie de página anterior se identificó hasta el momento el origen europeo de uno de los cuerpos

${ }^{8}$ Por pedido de las Hermanas de María Auxiliadora se recuperaron, estudiaron y trasladaron los restos de dos Hermanas enterradas en el cementerio y que era posible identificar sutumbas (Suby y colaboradores 2009).
}

Figura 1). Este modelo trata de representar (de una forma simple) ideas que subyacen a algunos posicionamientos. Se parte de que la comunidades de Investigadores $(\mathrm{CI})$ deben generar información (Conocimiento científico objetivo CCO). Las comunidades educativas (CE) toman esos conocimientos y los transmiten a la sus Alumnos. Por último las comunidades originarias (CO) incorporan los CCO en el "marco de ser parte de la sociedad". En caso de que exista algún tipo de conflicto la comunidad Judicial (CJ) y la comunidad de Aplicación (Municipal, provincial o nacional) pueden desplegar mecanismos legales y determinará quienes se ajustan a las normas establecidas (tienen "la verdad jurídica") y quienes no se ajustan. En todos los casos se entiende que estos conforman los ámbitos en donde se encuentran los procedimientos adecuados y establecidos para "resolver conflictos". Esto se da en el marco de un paraguas de identidad nacional (históricamente en Argentina la educación tuvo como uno de sus objetivos homogenizar) que es la que legitima simbólicamente esta linealidad ${ }^{9}$ (Rodríguez y colaboradores 2016).

Dentro de la comunidad científica $(\mathrm{Cl})$ podemos encontrar diferentes posiciones respecto a la neutralidad de ciencia. Giardina (2001) trae el ejemplo del martillo que creo puede ser útil en para nuestro caso. Se ha planteado que el martillo

\begin{abstract}
"... puede servir tanto para el útil, sano y natural fin de clavar clavos como satisfacer la brutal intención de partirle la cabeza al próximo (...) el uso que se le asigne cada vez a la herramienta ya no será una cuestión que dependa del conocimiento que originó la herramienta martillo y, menos, por supuesto, del martillo mismo (...) habrá que buscar las responsabilidades en la intención de quien se sirve del martillo desnaturalizando el fin para el que fue concebido..." (Giardina 2001:350).
\end{abstract}

Siguiendo este esquema se puede considerar que si los arqueólogos y biantropólogos generamos conocimientos científicos objetivos, entonces su uso no depende de nosotros. Alguien podría plantear desde este "marco neutral" que si aparecen problemas estos no tiene ninguna relación con esta manera de entender "la ciencia" (en nuestro caso la Arqueología y Bioantropología). Pregunta ¿El problema entonces sería de "la realidad" si no se ajusta a esta manera de entender la ciencia?

En este modelo lineal simplista tal como fue presentado, las "verdades", la "objetividad de la ciencia", así como las responsabilidades de cada comunidad no cambian. Uno de los problemas a resolver sería cómo incluir otros aspectos: los cambios históricos y la relatividad de las

\footnotetext{
${ }^{9}$ El no reconocimiento en algunos casos del estado nación por parte de las CO rompen con esta linealidad. No creemos que este sea el único caso donde la linealidad es interpelada.
} 
verdades por ejemplo. Como entonces dar cuenta de la temporalidad como parte del conjunto de cuestiones que aquí englobaremos dentro de la palabra contexto. Podríamos sugerir entonces generar gráficos como el de la figura 1 para cada momento histórico. Se trata de un buen ajuste al modelo pero nos preguntamos si con eso alcanza.

\section{Judicialización y otras formas de abordar los conflictos}

Muchos conflictos (entre ellos los de $\mathrm{CO}$ y $\mathrm{Cl}$ ) suelen tratarse en contextos judiciales. Esto presupone que el derecho (la ley) es capaz de abordar con un sentido de "justicia" los conflictos sociales.La relación entre Ley y Hecho es compleja. Ante las dificultades de las normas para considerar el cambio Geertz (1994) plantea la necesidad de que la comunidad judicial se involucre (sensibilidad legal) en la busca de formas creativas para enfrentar situaciones nuevas. Nato y col. (2006) plantean que el de alguna forma el Derecho "esencializa". Asimismo estos autores sugieren que, sin descartar su rol en nuestra sociedad actual, los conflictos sociales son fenómenos complejos y que la lógica del derecho no fue concebido para su atención..." más adelante señalan que el "...derecho es un instrumento que se puede utilizar para ciertos fines, pero no como un elemento autónomo sino como parte de un conjunto de instrumentos posibles..." (Nató et al 2006:121). En este sentido (no en todas las situaciones), incorporan como parte de las herramientas disponibles otras estrategias cómo por ejemplo la Mediación Cultural en sus distintas formas. Por tanto debemos considerar que quizás el accionar del sistema judicial no alcanzar en todos los casos para tratar conflictos sociales complejos como los de $\mathrm{CO}$ y $\mathrm{Cl}$.

En relación a la Mediación ${ }^{10}$ hay algunos aspectos que llamaron mi atención. Las prácticas tradicionales del sistema judicial están interesadas en resolver si $A$ o $B$ tienen razón. En las prácticas de mediación interesa por sobre todoatender el cuidado de los vínculos entre las personas involucradas. Por otra parte los aspectos relacionados al poder que cada una las partes tiene en el conflicto no ayuda y tiende a complicar el problema. Los interesados son participes activos de la solución y se trabaja para que puedan poner sobre la mesa sus intereses como parte de los caminos para gestionar o transitar el conflicto (Nato y colaboradores. 2006). Los arqueólogos y bioantropólogos no podemos ser mediadores porque

\footnotetext{
10 Matta define a la mediación: "...Esta modalidad sintetiza un complejo proceso sociocultural que, ante la creciente demanda de acceso a justicia, busca redefinir focos de juridicidad (Garapon 1997). Enmarcada en los denominados métodos alternativos de resolución de conflictos (Cappelletti 1993) o justicia restaurativa (Llobet 2005), y frente a las formas tradicionales de tratamiento de la conflictividad ciudadana, la mediación comunitaria se organiza, y se enmarca, en un tipo singular de sensibilidad legal (Geertz 1994). Este dispositivo se encuadra en la noción más amplia de justicia restaurativa -el conflicto es entre partes-, que se opone a la concepción tradicional de justicia retributiva -el conflicto es con la norma y se penaliza(Dignan 2005; Llobet 2005)..." (en Matta 2016:58).
}

tenemos nuestros intereses y en el mejor de los casos podríamos ser facilitadores. Por último,como señala Lederach en su experiencia, no todos los conflictos se resuelven con la presencia de mediadores, aunque como veremos más adelante Lederech encuentran patrones en todos los casos (Lederach 2006).

Utilizaremos nuevamente el tema de la neutralidad para plantear otra "cara" de esta problemática. Nos preguntamos ¿Es posible desde una posición neutral y objetiva al margen de la sociedad, sus intereses y conflictos llevar adelante restituciones de restos humanos a las comunidades originarias que las reclaman? Mancisidor (2014) plantea el derecho de la ciencia, y la Ley Nacional 25.517(2001), reglamentada por el Decreto 701/2010sostiene entre otros aspectos los derechos de las comunidades originarias. El Código de ética profesional en arqueología aborda estos punto (Aranda y colaboradores, 2014) y las complejas instancias que implican los procesos de restitución son motivos de trabajo en diferentes ámbitos como los talleres de restituciones que se vienen realizando en Argentina. Entre sus considerandos el código de Ética de la American Asociation of Physical Anthropology (AAPA 2003) señala la necesidad de trabajar en todos los ámbitos ${ }^{11}$ en marcos (no formulas) que ayuden a la toma de decisiones que en cada caso es particular. Ningún código de ética puede anticipar circunstancias únicas o acciones directas requeridas en cualquier situación específica.

\section{Actitudes frente al conflicto}

Por otra parte Markus y Mc Cormack (en Nató y colaboradores 2006) proponen (cómo una primera aproximación) que las actitudes de los diferentes actores frente al conflicto pueden ubicarse en un eje cartesiano donde se consideran dos variables niveles de competencia (necesidad de satisfacer los propios intereses) y niveles de colaboración (posibilidad de satisfacer los intereses de la otra persona). En estos ejes (ver figura 2) podemos ubicar actitudes extremas orientadas a aceptar y acomodarse contrapuestas con otras orientadas a competir y enfrentar. También en el gráfico planteado por los autores es posible observar una diagonal que va desde evitar contender, pasando por acordar y contemporizar, hasta colaborar y desarrollar. Este esquema resulta didáctico porque parece incluir un amplio abanico de actitudes y donde los diferentes actores pueden sentirse más o menos representados en sus actitudes en estos espacios de intereses diferentes.

Por otra parte cómo señala Morín (Morín 1977 en García 2006) la complejidad es la imposibilidad de simplificar. Y esto último aparece como uno los cuatro patrones y dinámicas que generan la complejidad de acuerdo con Lederach (2006). Concretamente este último autor señala que debemos considerar que los "conflictos sociales complejos" requieren tanto para el análisis como para la

\footnotetext{
${ }^{11}$ Incluida la formación de grado.
} 


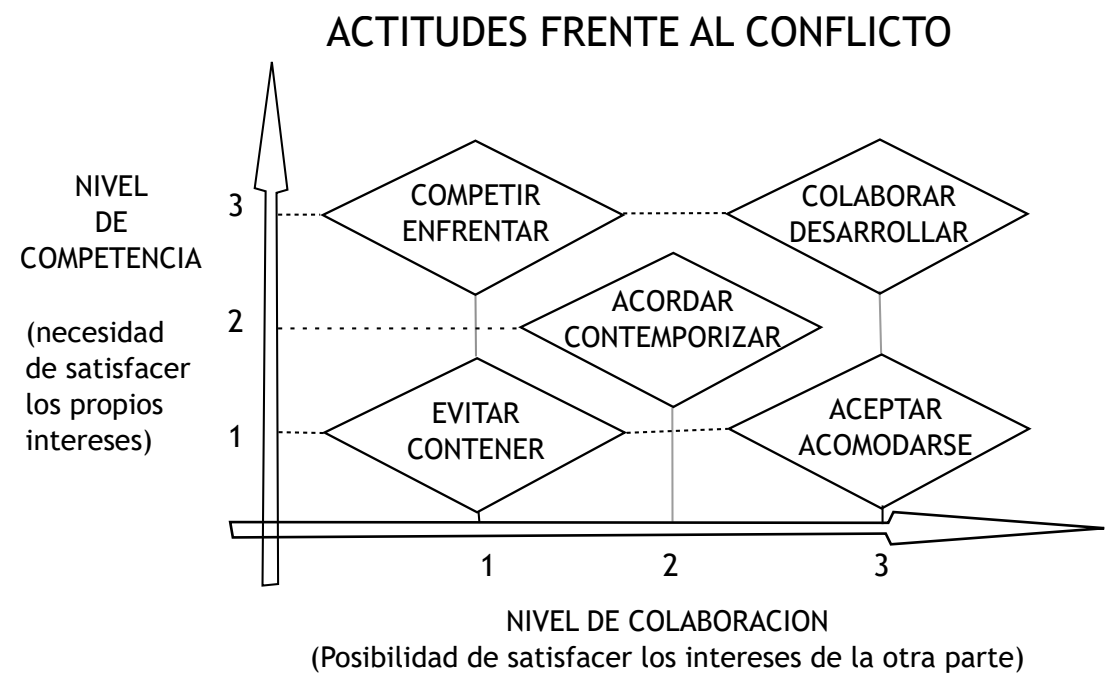

Figura 2. Actitudes frente al conflicto. Extraído de Natóet al 2006 y elaborado originalmente por Markus y Mc Cormack.

Figure 2: Attitudes in conflict situations. Extratacted from Nató et al 2006 originally by Markus and Mc Cormack.

acción de "La práctica de la curiosidad fuera de lo que denomina las polarizaciones duales (blancos-negros).

En este punto creo oportuno traer a Lederach (2006) y su reflexión sobre el realismo y el pesimismo. Claro que es posible pensar que los cambios no son fáciles ni rápidos. Este autor señala frente a los conflictos sociales complejos que no debemos considerar los cambios en términos de meses o un año, el cambio señala necesita décadas, e incluso generaciones.Es interesante su reflexión sobre el pesimismo al señalar que proporciona un punto de partida para comprender la naturaleza del cambio. Los cambios dice Lederach deben contemplar la complejidad y plantearse un compromiso a largo plazo. El plantea

"...No estamos hablando aquí de una actitud nacida del cinismo, de una actitud resentida y una predisposición a ver lo peor de todo y de todas las personas, la búsqueda de fallos por excelencia. El pesimismo nacido del cinismo es una lujosa evitación del compromiso. El pesimismo del que hablamos surge de la experiencia tenazmente adquirida, como guía que pone a prueba la calidad del compromiso..." (Lederchar 2006:96 las negritas son nuestras).

Asimismo para no generar falsas expectativas este autor nos dice

"...los acuerdos a que se puedan llegar en la mayoría de los casos no son soluciones, son propuestas para procesos negociados, que, si se producen, cambiarán la expresión del conflicto y suministrarán cauces para redefinir las relaciones..."(Lederach 2006:85 las negritas son nuestras).
Pero como propone Lederach allí debe estar nuestra imaginación (el arte de crear lo que no existe) como camino.

\section{Modelo no lineal de relación entre $\mathrm{CO}$ y $\mathrm{Cl}$}

Del diálogo, antes mencionado (entre nuestras experiencias personales y una serie de lecturas) recuperamos un nombre para caracterizar las relaciones entre miembros de $\mathrm{CO}$ y Cl "conflictos sociales complejos". Siguiendo a Lederach definimos conflictos sociales complejos como aquellos donde "... múltiples actores persiguen múltiples acciones e iniciativas, al mismo tiempo, en numerosos niveles de relaciones sociales en un escenario interdependiente..." (Lederach 2006:68) en un lugar determinado. Aquí no hay linealidad e incorporamos la idea de lugar de Geertz (1994).

Estando parados frente al mismo problema con que iniciamos este camino (la relación entre comunidades originarias y científicas) este modelo no lineal (figura 3) nos enfrenta a otro escenario.

\section{La coordinación e integración de enfoques como problema}

¿Cómo ingresar a este escenario no lineal de la figura 3 ? No hay caminos. Nuestro recorrido con toda su impronta particular y local fue a los tumbos, en forma intuitiva. En el camino una serie voces (textos) que fuimos encontrando nos ayudaron a darle claridad y algún grado de formalización a lo que estábamos viviendo y pensado.

Retomando a Lederech la complejidad de los conflictos sociales emerge de la multiplicidad, interdependencia y simultaneidad" (Lederach 2006) decíamos. A este tipo de problemáticas García (2006) las concibe como "sistemas 


\section{Originarias (CO)}

\section{C. de Aplicación (CA)}

Municipales,
Provinciales y
Nacionales

\section{Judiciales (CJ) \\ Provinciales y Nacionales}

Figura 3: Relación entre Comunidades (Investigadores, Educativa, Originarias y otros actores sociales). Modelo de la complejidad.

Figure 3: Relationship between Communities (Researchers, Education, and other social actors) Complex model. complejos" cuyos elementos están interdefinidos y cuyo "estudio" requiere de la coordinación de enfoques disciplinarios que deben ser integrados en un enfoque común. De ahí el autor señala que la interdisciplina implica el estudio de problemáticas concebidas como sistemas complejos. En este sentido puede ser importante reflexionar sobre el tipo de abordaje $\mathrm{e}^{12}$ (disciplinar, multidisciplinar, inter-disciplinar y trans- disciplinar) para tratar las relaciones entre miembros de $\mathrm{CO}$ y $\mathrm{Cl}$ en términos de "conflictos sociales complejos".

García agrega que

"... La delimitación de un sistema complejo no sólo requiere de una concepción común entre los miembros del equipo sobre la problemática general a estudiar, sino también de una base conceptual común y de una concepción compartida de la investigación científica y de sus relaciones con la sociedad..." (Rolando García 2006:34).

Considerando la perspectiva de García (op cit) la coordinación de enfoques, concepción y base

\footnotetext{
12 Cómo una primera aproximaciones importante aclarar qué entendemos como estrategias de abordaje disciplinar, multi-disciplinar, inter-disciplinar y trans- disciplinar (re-elaborado de García 2006). Por disciplinar se podría definir como el trabajo desde una disciplina que desarrolla una metodología, genera una serie de presupuestos o toma información ya generada de otras líneas, y realiza inferencias. En la perspectiva multidisciplinar sumamos los resultados de investigaciones parciales realizadas en forma independiente y al final elaboramos una interpretación que incluye de alguna forma toda la información obtenida. En el caso de la inter-disciplina los especialistas de diferentes líneas parten de un problema que definen como abordable desde sus líneas de trabajo. Luego establecen formas de complementariedad a priori entre los datos de cada línea. A lo largo del proyecto se reúnen y a partir del trabajo específico en territorio redefinen el problema en espacios de diálogo continuo. Por último la perspectiva trans disciplinar la pensamos como similar al inter disciplina pero incorporando actores y miradas no vinculadas al área científica y relevante para el problema definido. Existen otras aproximaciones a estos términos como la señalada por Sotolongo (2010) que especialmente en la idea de transdiciplinar incorporar otros aspectos o miradas sumamente interesantes.
}

C. Científicas (Cl) conceptual común tanto dentro de cada comunidad como entre comunidades nos enfrenta con varios problemas para los cuales no tenemos respuesta. ¿Qué ocurre cuando dentro de la comunidad de investigadores las diferentes líneas de investigación parten de diferentes marcos teóricos (procesuales y post procesuales por ejemplo en el caso de la Arqueología). ¿Es posible la coordinación de enfoques y generación de una base conceptual común en estos casos? O quizás sea más "prudente" en un estudio interdisciplinario descartar la posibilidad de coordinación de marcos teóricos que parten de supuestos muy diferentes. ¿Esto implica que los estudios interdisciplinarios únicamente deben coordinar estudios dentro de marcos compatibles? Como alternativa a lo recién planteado: ¿podríamos partir de que la articulación entre marcos Procesual y Postprocesual en Arqueología es un supuesto operativo (sin analizarlo) y que esto no tendrá "consecuencias"?

Por otra parte si consideramos que los conflictos sociales complejos son contradictorios internamente entonces ¿necesitamos de una base conceptual común(en términos de García 2006) o ¿podemos operar con marcos teóricos contradictorios entre sí? En relación a esto último si la configuración de "puentes" entre marcos teóricos u otras aproximaciones fuera posible ¿cuáles podrían ser los problemas que estosplantean? No tenemos respuestas a estar preguntas pero si el interés por aproximarnos desde perspectivas que contemplen la complejidad y explorar los espacios de interacción entre diferentes miradas.

\section{Espacios y diálogo y procesos}

En el punto anterior hicimos especial referencia a la coordinación de enfoques y tenciones dentro de la comunidad científica y con otras. Las comunidades originarias también enfrentan en algunos casos desafíos vinculados a estos aspectos a su interior. En relación a esto son los miembros de estas comunidades lo que (si lo consideran oportuno y necesario) hablaran. 
Una de las dimensiones de las relaciones entre comunidades originarias y científicas ${ }^{13}$ la concepción del tiempo ${ }^{14}$ de arribo a una redefinición de las relaciones o a consensos pueden generar desencuentros. Concretamente nuestros cronogramas de proyectos de investigación científica generan tensiones internas y externas.

Por otra parte, el Encuentro Nacional de Organizaciones Territoriales de Pueblos Originarios (ENOTPO) generó un "Protocolo de Consentimiento Previo Libre Informado (2014). De este texto nos interesa destacar dos cuestiones: la idea de proceso y dentro del mismo de etapas de diálogo intercultural. Estos dos aspectos no descartan las tensiones sin embargo aparecen como posibilitadores, que ayudan, previenen y ponen en el centro los vínculos.

En relación a esto último el Protocolo de la comunidad Miskitu de Honduras del $2012^{15}$ plantea siete pasos interesantes en el marco de un proceso de consentimiento libre informado: 1) primer contacto; 2) acuerdo sobre el proceso; 3) discusión de información relevante; 4) toma de decisión; 5) negociación entre comunidades y actores relevantes; 6) acuerdo sobre consentimiento; y 7) implementación y monitoreo.

Un aspecto interesante del documento Mosquita es que plantea la realización de talleres orientados a discutir y analizar las siguientes preguntas: ¿Quiénes son los actores que deben ser consultados Incluidos mujeres, jóvenes, ancianos? ¿QQuiénes podrán dar el consentimiento en representación de sus bases?; c) ¿Quién o quiénes pueden actuar como observadores del proceso de consulta?; d) ¿Con cuánto tiempo de anticipación deben socializarse los contenidos de la consulta?; e) ¿Qué medios de comunicación son los más apropiados para la convocatoria y comunicar acuerdos?; f) ¿Cómo podemos regionalizar la consulta y devolución de resultados?; ¿Cómo debemos abordar a la población no originaria en territorios de población originaria? Y ¿Existe algún procedimiento que prefieran para proceder con la consulta?

Creemos que tanto el primer paso planteado por este protocolo vinculado a "primer contacto" como las preguntas acompañan la idea de que los procesos son

\footnotetext{
13 Las relaciones del tipo víctima-victimario han formado parte de la historia entre $\mathrm{CO}$ y $\mathrm{Cl}$ y su reconocimiento es relevante así como también la construcción de nuevas formas de relación.

${ }^{14}$ No es tema de este trabajo pero, tampoco podemos asumir que otros aspectos como la concepción de la propiedad y la relación con la naturaleza sea la misma.

15 Este documento fue desarrollado por MASTA (Mosquitia Asla Takanka-Unidad de la Mosquitia. Con la asesoría técnica de Janina Heim, pasante para la Unión Internacional para la Conservación de la Naturaleza (UICN) Olman Varela y Ronald McCarthy, Oficina Regional para Mesoamérica y la Iniciativa Caribe, UICN. Centro de Derecho Ambiental, UICN, Bonn, Alemania. Johanna Von Braun, Justicia Natural.Con el apoyo del Ministerio Federal de Cooperación Económica y Desarrollo (BMZ). UICN Octubre, 2012 Honduras.
}

Iocales y difícilmente pueden llegar a éxito planteado con criterios de homogeneidad para todas las comunidades de un territorio tan amplio como la Argentina.

En Argentina nuestras asociaciones profesionales y muchos colegas están trabajando en diferentes aspectos vinculados a esta problemática y también en espacios de diálogo. Sin pretender hacer un análisis exhaustivo podemos mencionar algunos. Las bases para la elaboración de un protocolo de consentimiento previo, libre e informado (CPLI) para el tratamiento de restos humanos. Este documento surgió inicialmente de la Jornada de Elaboración del Proyecto de Protocolo Base de Tratamiento de Restos Humanos (Instituto Nacional de Antropología y Pensamiento Latinoamericano INAPL, ciudad autónoma de Buenos Aires, 27 de septiembre de 2013). Se efectuaron modificaciones en el año 2014 por nuevas sugerencias de socios y representante del Museo de Ciencias Naturales de ciudad de La Plata. Luego, esta versión fue revisada de acuerdo con las propuestas del V Taller de Discusión sobre Restitución de restos humanos de interés Arqueológico y Bioantropológico-TaDIRh- organizado por IDACORCONICET, Museo de Antropología (Facultad de Filosofía y Humanidades, Universidad Nacional de Córdoba, 2015) por representantes de la Asociación de Arqueólogos Profesionales de la República Argentina (AAPRA), de la Sociedad Argentina de Antropología (SAA), del Museo Etnográfico "JB Ambrosetti", Facultad de Filosofía y Letras de la UBA y profesionales asistentes a la reunión convocada por la AAPRA de agosto 2015. Asimismo es importante mencionar el Proyecto de Protocolo Base para el desarrollo de investigaciones científicas y tratamiento de restos humanos de interés científico en el marco de la aplicación de la Ley 25.517 y el Decreto 701/10.

En nuestra experiencia personal y profesional nuestras primeras vinculaciones con miembros de comunidades surgen en relación a nuestro interés por estudiar restos óseos de personas que sabíamos en algunos casos que podían ser familiares de alguno de ellos. Partimos de considerar que los restos humanos son una parte muy especial del pasado. En el marco de la "plataforma de trabajo" (ver pié de página 2) acordamos que debíamos avanzar en estrategias donde los "restos humanos no sean considerados como objetos sino como personas" (Nahuelquir y colaboradores 2015 y Guichón y colaboradores. 2015).

Puesto en palabras de alguna forma fuimos construyendo entre todos los actores en Puerto Santa Cruz y en el Norte de Tierra del Fuego algunas cuestiones que podrían explicitarse en los siguientes términos:

- $\quad$ El pasado puede ser espacio de conflicto de intereses y su resolución depende de nuestra capacidad de diálogo.

- Nos interesa mantener los vínculos entre las personas que participan de este diálogo. 
- $\quad$ Estamos dispuestos a ponernos en el lugar del otro. ${ }^{16}$

El diálogo tal como lo estamos pensando y vivenciando implica un proceso que incluye la diferenciación de los actores desde sus perspectivas y algún grado variable de integración (o reintegración) en el marco de la realidad de la que todos formamos parte. Este diálogo, en nuestro caso particular, contempla que los actores están dispuestos explícita o implícitamente a considerar algún grado de incompletitud en las cosmovisiones de cada uno en relación al problema en cuestión. Como señala Boanventura de Sousa Santos (2002) todas las culturas son incompletas en su concepción de la dignidad humana. El resultado se espera que sea una totalidad conceptualmente más enriquecida. En alguna medida la interculturalidad tiene algún parecido con la interdisciplina. Repensando las ideas de García (2006) en una perspectiva más amplia es posible señalar que

\section{"...la acción conjunta...en busca de respuestas comunes a problemas que inicialmente pueden ser considerados distintos por sus orígenes sociales, físicos o biológicos, plantea nuevas situaciones metodológicas y conceptuales..."}

En ello hay implícito un gran esfuerzo por reconciliar en cada momento unidad y diversidad, especialidad y universalidad (Najmanovich 2002). La "toma de distancia" que los actores deben realizar con respecto a los objetivos e intereses particulares (disciplinar o de otro tipo), significa una apertura a métodos (caminos), conceptos y lenguajes poco familiares. Se trata, sobre todo, de una búsqueda constante, desde cada espacio disciplinar, cosmovisión o normativo. Esto incluye también las relaciones y los vínculos ${ }^{17}$ Najmanovich (2002).

\section{A modo de conclusión}

Somos parte de un conjunto de personas (miembros de comunidades originarias e investigadores) que estamos transitando lo que podríamos considerar como "conflictos sociales complejos". En forma esquemática en este trabajo se sugirió como personas de diferentes comunidades científicas, originarias, educativas, judicial y de organismos de aplicación, podrían gestionar sus conflictos partiendo de un modelo lineal simplista. Por

\footnotetext{
${ }_{16}$ Cabe señalar que esto implicó beneficiarnos como científicos de la retroalimentación que esa interacción implica. Como un ejemplo ambas comunidades $(\mathrm{Cl}$ y $\mathrm{CO}$ ) pueden coincidir contra decisiones que afectan intereses mutuos como las actividades de coleccionistas, mineras, o el Rally Dakar.

${ }^{17}$ Como señaló uno de los evaluadores anónimos de este trabajo “....En este punto, se ponen en juego tanto los contextos históricos como las trayectorias sociales de los grupos, y por ello la particularidad de cada caso no sólo se piensa a partir de la demanda efectiva formulada por la CO, sino también por los sentidos particulares derivados de la(s) lectura(s) histórica(s) del grupo ".
}

otra parte, por medio del trabajo de Nato y colaboradores (2006) recuperamos la necesidad de satisfacer intereses propios y la posibilidad de considerar los de otros visualizando niveles de competencia y colaboración de que entendemos permite ubicar diferentes posicionamientos de ambas comunidades.

En este punto la necesidad de repensar la idea de objetividad, las verdades absolutas, y la inclusión de otras dimensiones como la histórica se mencionan en términos de preguntas abiertas.

Se plantea que la judicialización de los conflictos entre comunidades originarias y científicas se enfrenta a grandes desafíos. Generar espacios locales y dinámicos que posibiliten el involucramiento de los actores a través de la mediación $u$ otros propuestas es una estrategia de apertura, de búsqueda de caminos a explorar. En este sentido rescatamos su carácter dinámico donde los procesos negociados como señala Lederech (2006) cambian la expresión del conflicto y suministran causes para redefinir las relaciones.

Los conflictos sociales complejos, como fue señalado emergen de la multiplicidad, interdependencia y simultaneidad, y la consideración para cada caso de los diferentes enfoques y cosmovisiones (en algunos casos contradictorios) parece requerir de miradas no lineales y simplistas sino perspectivas complejas. La importancia de los vínculos entre los actores y de disposición para tiempos largos parecen ser aspectos que abren posibilidades.

Lederech (2006) sugiere cuatro patrones y dinámicas que generan la complejidad. La primera la centralidad de las relaciones ${ }^{18}$. Un aspecto contextual que tiene que ver con nuestra práctica y vivencia diaria es que en general la comunidad de investigadores somos rentados por el estado con dedicación para atender estos temas. Si el objetivo es desarrollar un diálogo intercultural nos preguntamos si no es necesario contar con actores de las comunidades originarias que desde su cosmovisión estén también sostenidos "económicamente" de alguna forma para disponer del tiempo y dedicación que requiere esta construcción de redes. Esto es especialmente importante si pensamos en prevención, monitoreo y prácticas educativas. Nuestra experiencia es que uno de los requerimientos básicos (no el único) para contar con la disponibilidad de cualquiera de nosotros para participar de espacios de diálogo es "tiempo".

Por otra parte la necesidad de trabajar en los grises y superar las interpretaciones duales (blanco o negro). El tercer aspecto es que en los conflictos sociales complejos no hay recetas. Hay que proponerse espacios

\footnotetext{
18 La centralidad de las relaciones de acuerdo con Lederach se vinculada con la construcción de Redes. En relación a este punto Najmanovich (2007) plantea que la concepción dinámica de redes es una estrategia para aproximarnos a la complejidad y presente doce interesantes puntos a considerar que a mi entender incorpora entre otras cosas "cambiar nuestra perspectiva".
} 
de búsqueda de formas creativas de abordarlos. Por último la voluntad de arriesgar. Una negociación o la búsqueda de formas de "gestionar" nos colocan en lugares donde tenemos que estar dispuestos a "perder" en alguna medida.

Por otra parte y en relación a los tiempos el cambio en muchos casos señala Lederech necesita décadas, e incluso generaciones (Lederech 2006).Teniendo en cuenta lo planteado quizás se puedan acordar objetivos intermedios mensurables en escalas más acotadas.

La elaboración de protocolos regionales consensuados entre investigadores, comunidades, autoridades locales provinciales y poder judicial sobre los procedimientos ante el hallazgo de restos óseos humanos con presunción que puedan pertenecer a contextos no judiciales es una necesidad (Fabra comunicación personal junto con otros colegas están desarrollando una experiencia muy interesante en la provincia de Córdoba). Estos protocolos deben incluir espacios de trabajo conjunto para ajustar procedimientos y generar estrategias orientadas a la prevención.

La complejidad siempre está presente ${ }^{19}$. La modernidad en muchos casos planteo a la simplificación, el análisis de parcialidades como estrategia para su abordaje. En nuestro caso la relación entre comunidades originarias y científicas donde multiplicidad, interdependencia y simultaneidad emergen como señala Lederach (2006) el desafió es encontrar perspectivas no lineales y simplistas para su abordaje. Lo aquí planteado podría ayudar a pensar algunas de las dimensiones posibles en el hacerpensar (Najmanovih comunicación personal) donde el involucramiento es clave. No hay recetas, tus caminos, mis caminos, nuestros caminos entendemos tienen muchas dimensiones siendo la local y temporal relevantes.

\section{Quequén, Necochea, 1 marzo del 2016}

\section{Agradecimientos}

Nuestro objetivo aquí fue poner en palabras escritas algunas cuestiones que vienen surgiendo en charlas en diferentes encuentros. Este manuscrito circuló previamente entre amigos y fue enriquecido con nuevas discusiones, intercambios y aclaraciones que lo mejoraron. En este sentido quiero agradecer por sus comentarios y sugerencias a María Teresa Fernández (compañera de más de 30 años), Juan Matta, Melisa Salerno, Sebastían Muñoz, Mariana Fabra y Luis Borrero. En este camino debo agradecer también la generosidad de compartir sus miradas y experiencias a Sergio Nahuelquier, Carlos Huilianio;

\footnotetext{
19 Algunos de los autores que desde perspectivas y temáticas diferentes aportan a la variedad de miradas sobre la complejidad. Entre 1940 y 1950 Bertanlanfly (teoría General de Sistemas); Beatson Rosembluet, Wiener von Foerster (Cibernética); Pitts y Mc Cullonc (Redes Neuronales). Entre 1960 y 1970 Mandelbrot (Geometría Fractal), Lorenz (Teoría del Caos), Prigogini (Termodinámica no lineal y procesos Irreversibles); Maturana y Varela (Autopoiesis); Ashby (Autoorganización); von Foerster (Cibernética de Segundo orden). Entre 1980 y 2010, Morín (Pensamiento complejo y filosofía de la complejidad) (extraído de Capra 1998 y Sotolongo 2010).
}

Rubén Maldonado; Brenda Biltle; Mirta y María Salamanca; Margarita Maldonado; Cristian Pantoja; Walter Barria y la Hermana Ana María Fernández. Sin las discusiones y el trabajo con mis colegas del Núcleo de Estudios Interdisciplinarios de Poblaciones Humanas de Patagonia Austral Pamela García Laborde, Josefina Motti, Marilina Martucci, Luciano Valenzuela, Manuel D’Angelo del Campo y Romina Casali nada sería posible. Las autoridades Salesianas tanto a nivel nacional como local de Río Grande facilitaron diferentes aspectos de este trabajo durante años. A las autoridades municipales de Río Grande (Tierra del Fuego) y de Puerto Santa Cruz (Pcia de Santa Cruz). A todos mis compañeros del equipo en Santa Cruz especialmente a Isabel Cruz (UNPA), Sebastián Muñoz y Jorge Suby. A los miembros de la Asociación Civil "Alfredo Marcenac" y de la Diplomatura en Educación para la Paz y el abordaje de la Conflictividad Social (FACSO-UNCPBA) con sede en Quequén por el espacio de aprendizaje en estos diez años de trabajo conjunto en temas de conflictividad social y complejidad. Agradezco a Denise Najmanovich, Pedro Sotolongo y todos mis compañeros del "Seminario Itinerarios de la Complejidad II" por haberme ayudado quizás sin saberlo en esta "travesía". Un especial agradecimiento a la Municipalidad de Puerto Santa Cruz, Parques Nacionales (Parque Nacional Monte León) y la Provincia de Tierra del Fuego. A la Familia Lemaire por su interés y respeto por el cuidado del pasado. Al Centro de Antiguos Pobladores de Río Grande, a la escuela Agrotécnica Salesiana de Río Grande, especialmente al padre Ticó y la Dr. Bitsch por su afecto, acompañamiento y charlas (ambos ya fallecidos). Al profesor Carlos Baldassarre que en diferentes momentos de su gestión en Río Grande confió en mi trabajo y criterio. Toda mi familia Grieco en Ushuaia fue y es un apoyo incondicional en cada viaje. Al CADIC-CONICET de Ushuaia y especialmente a Mónica Salemme, Martín Vázquez y Fernando Santiago por su ayuda y apoyo. A la Municipalidad de Río Grande, a los directivos y el personal del Museo del Fin del Mundo. Estas actividades se desarrollan en el marco de proyectos PIP/CONICET 112-200801-00996, UNPA 29/A206-1, 05/F723 SeCyT-UNC y UBACyT 20020090200015; Los proyectos Secyt son el 05/F723 y el 162/12; UBACYt F447; PIP 5576; PICT 01520 y PICT 0575.

\section{Bibliografía}

AAPA, 2003. Code of Ethics of the American Association of Physical Anthropologists. Approved by the AAPA Membership at the annual business meeting on April 25. 2003. http://www. physanth.org/positions/ethics.htm (última consulta 2/03/2016)

Aranda, C., G.Barrientos, y M.C. Del Papa 2014 Código Deontológico para el estudio, conservación y gestión de restos humanos de poblaciones del pasado. Revista Argentina de Antropología Biológica, Vol. 16 (2): 111-113, Julio Diciembre. Argentina.

Capra, F. 1998 La trama de la vida. Una nueva perspectiva de los sistemas vivos. Editado por ANAGRAMA, BARCELONA. España.

De Sousa Santos, B. 2002 Hacia una concepción multicultural de los derechos humanos. En el Otro Derecho, N 28: julio:59-83. ILSA, Bogota, DC. Colombia.

ENOTPO 2014 Protocolo de Consulta Previa, Libre e Informada a Pueblos Originales. 48 pp. https//drive.google.com/file/ d/OB7mfpufuTf3UWjdRQzdnZFpoc28/edit 
García R. 2006 Sistemas complejos Conceptos, método y fundamentación epistemológica de la investigación interdisciplinaria Editorial Gedisa, S.A.) Barcelona, España pp 2026 capítulos y conclusiones (Última fecha de consulta) 2/3/2016. (http://www.pensamientocomplejo.com.ar/docs/ files/Garcia,\%20Rolando\%20-\%20Sistemas\%20Complejos. $p d f$.

Geertz C-1994 Conocimiento Local, Ensayos sobre la interpretación de las culturas. Ediciones Paidos, Impreso en España-297 pp.

Giardina M. 2000 El problema de la Neutralidad en Ciencia y Tecnología, E 349-368. En La Posciencia, el conocimiento científico en las postrimerías de la modernidad Editora Esther Díaz, Editorial Biblos (tercera Edición). Buenos Aires, Argentina.

Guichón RA; R Casali; P García Laborde; MA Salerno y R Guichón Fernandez. 2016 (ep) "An interdisciplinary approach to coloniality in Tierra del Fuego (late 19th - early 20th centuries)" Capítulo 4 del libro Bioarchaeology of Contact, Colonial Encounters and Colonialism Edited by Melissa S. Murphy and Haagen D. Klaus. Edit. University Press of Florida bioarchaeology series (Bioarchaeological Interpretations of the Human Past: Local, Regional, and Global Perspectives). http://www.upf.com/ seriesresult.asp?ser=bioarc.

Guichón R.A, P. García Laborde, J.M.B. Motti, M. Martucci, R. Casali, F. Huilinao, M. Maldonado, M. Salamanca, B. Bilte, A. Guevara, C.G. Gallardo Pantoja, M.A. Suarez, M.A. Salerno, L.O. Valenzuela, M.D. D'Angelo del Campo y P.I. Palacio 2015 Experiencias de trabajo conjunto entre investigadores y pueblos originarios. El caso de Patagonia Austral. Revista Argentina de Antropología Biológica Volumen 17, número 2, páginas 00-00. Julio-diciembre 2015 (aceptado para su publicación).

Lederech J.P. 2006 La Imaginación Moral: El Arte y el Alma de la Construcción de la Paz. Editoral Bakeaz Centro Documentación para la Paz. 267 pp. Bilbao, España.

Ley 255172001 Nación: Disposición sobre restos mortales de Aborígenes que formen parte del Museos y/o colecciones públicas o privadas. En www.sinca.gob.ar/sic/gestion/ legislacion/ley.php?id=377.

Ley 25517 y Decreto 7012010 reglamentación Consultado en https://restituciondecuerpos.wordpress.com/ley-nacional-255172001-y-su-decreto-reglamentario-7012010/.

Ley 2785 Adhesión a la Ley Nacional 23302 de la Provincia de Santa Cruz. www.santacruz.gov.ar/derechos/index. php?opcion=ley2785.

Mancisidor, M. 2014 El derecho a la ciencia, tan necesario como desconocido. Foro Científico. Investigación y Ciencia. Noviembre: pagina 44. Edición Española de Scientific American.

Matta, J. P. 2016 Entre vecinos eso no se hace. Sentidos de justicia y de vecindad en el marco de un dispositivo institucional de administración de conflictos. Antípoda. Revista de Antropología y Arqueología, núm. 24, enero-abril, 2016, pp. 55-71 Universidad de Los Andes Bogotá, Colombia.

Maturana, H.R. y F. Varela G. 1993 El árbol del Conocimiento. Colección Fuera de Serie, Editorial Universitaria 172pp. Santiago de Chile, Chile.
Morín, E. 1993 El Método l: La naturaleza de la naturaleza. Madrid: Cátedra.

Morín E. 2002 La cabeza bien puesta. Repensar la reforma. Reformar el pensamiento. Primera Edición 5 reimpresión, Buenos Aires, Argentina.143pp.

Nahuelquir, S. C.Huiliano, F.Huillino, R.A. Guichón, S.Caracotche y P. García Laborde 2015 Trabajamos juntos. Antes y después de la ordenanza municipal de puerto santa cruz 169/09. En "La arqueología pública en Argentina: historias, tendencias y desafíos en la construcción de un campo disciplinar" Editado por Mariana Fabra ; Mónica Montenegro ; Mariela Eleonora Zabala. - 1a ed. - San Salvador de Jujuy : Editorial de la Universidad Nacional de Jujuy - EDIUNJU. Facultad de Humanidades y Ciencias Sociales. , 2014. 320p; $24 \times 18 \mathrm{~cm}$. (Pasado Pensado - Serie Huellas / Mónica Montenegro) ISBN 978-950-721-481-3.

Najmanovich, D. 2002. From paradigms to figures of thought, Revista Emergence: Complexity and Organization Volume 4 Numbers 1 \& 2: 85-92. https://www.researchgate.net/ publication/255595178.

Najmanovich, D. 2007 El desafío de la Complejidad: Redes, cartografías dinámicas y mundos implicados. Utopía y Praxis Latinoamericana / Año 12. № 38 (Julio-Septiembre, 2007) Pp. 71 - 82 Revista Internacional de Filosofía Iberoamericana y Teoría Social / ISSN 1315-5216 CESA - FACES - Universidad del Zulia. Maracaibo-Venezuela.

Nató, A.M. Rodríguez Querejazu, M.G. Carbajal L.M. 2006. Mediación comunitaria. Buenos Aires. Universidad, 240 p, ISBN 950-679-392-1 1.

Protocolo Bio-cultural del Pueblo Indígena Miskitu El derecho al consentimiento libre, previo e informado en nuestro territorio de La Muskitia Hondureña 2012 https://cmsdata.iucn.org/ downloads/protocolo_miskitu.pdf(Última Consulta 2/03/2016).

Rappaport J. 2007 Más allá de la escritura: la epistemología de la etnografía en colaboración. Revista Colombiana de Antropología, vol43: enero-diciembre, pp: 197-229. Instituto Colombiano de Antropología e Historia. Colombia.

Restrepo, E. y A. Escobar 2005 "Other anthropologies and anthropology otherwise": Steps to a world anthropologies framework. Critique of Anthropology, 25 (2): 99-129. Durham: Duke University Press.

Rodríguez, M.E. L Horlent y colaboradores tehuelches y selk'nam. 201612 Tehuelches y Selk'nam: "No desaparecimos", En Pueblos Indígenas en la Argentina, historias, culturas, lenguas y educación. Editado por Ministerio de Educación y Deporte de la Nación. 36 páginas. Argentina.

Sotolongo, P.L. 2010 El pensamiento y las ciencias de "la complejidad" y la comunicación. Quórum Académico, Vol 7 N1 enero junio: 119-140. Universidad de Zulia Venezuela.

Suby, J.A. R.A. Guichón y A. Ibañez 2009. The Sisters Of The End Of The World. The Health Of The Salesian Sisters In The Mission "La Candelaria" (Tierra Del Fuego-Argentina). Libro de resúmenes: 111- III Paleopathology Association Meeting in South America. Necochea, 14 al 16 de Octubre de 2009. Argentina. 